\title{
Cholinergic modulation for hippocampal long-term potentiation
}

\author{
ETSUKO SUZUKI ${ }^{1}$
}

\begin{abstract}
Long-lasting change of the synaptic efficacy of neurotransmission in the hippocampus is thought to be one of the physiological bases of memory. Acetylcholine, one of the neurotransmitters, is mainly released from the medial septum to the hippocampus. The activation of muscarinic acetylcholine receptors (mAChR) reportedly enhances hippocampal synaptic plasticity; however, the mechanism by which it does this remains unclear. We addressed the involvement of the inhibition of $\mathrm{Kv} 7 / \mathrm{M} \mathrm{K}^{+}$channels, which are a target of mAChR modulation, during $\mathrm{mAChR}$ activation-induced enhancement of long-term potentiation (LTP) at rat hippocampal Schaffer collateral (SC)-CA1 synapses.
\end{abstract}

Key words : acetylcholine, potassium channel, hippocampal plasticity

Hippocampal synaptic plasticity has been considered to be one of the physiological bases of memory (Bliss \& Collinglidge, 1993). This plasticity is mainly mediated by glutamate, the main excitatory neurotransmitter in the hippocampus. At Schaffer collateral (SC)-CA1 synapses, AMPA-type glutamate receptors (AMPA receptors) receive presynaptically released glutamate. When postsynaptic membranes are strongly activated (depolarized) by an intense stimulus, such as highfrequency stimulation (e.g. tetanic stimulation), NMDA-type glutamate receptors (NMDA receptors) are activated, which results in the influx of $\mathrm{Ca}^{2+}$. The influx of $\mathrm{Ca}^{2+}$ activates intracellular second messengers (e.g. CaMKII), and then the number of AMPA receptors at the membrane surface increases and they become phosphorylated. After that, the response to the released glutamate is enhanced due to the changes of the AMPA receptors. This long-lasting enhancement of

1) Dept. Neurobiology, Grad. Sch. Med. Hokkaido Univ., JSPS Research Fellow

EN5-107, North 15, West 7, Kita-ku, Sapporo, 060-8638, JAPAN

Corresponding author: ETSUKO SUZUKI

E-mail: esuzuki@med.hokudai.ac.jp

J-STAGE Advance Published Date: June 16, 2014. doi: $10.2502 /$ janip.64.1.4 the efficacy of neurotransmission is called long-term potentiation (LTP).

In the hippocampus, acetylcholine, one of the neurotransmitters, is mainly released from the fibers originating from the medial septum. It has been reported that acetylcholine-related enzymes are reduced in Alzheimer's disease patients (Davies \& Maloney, 1976; Geula \& Mesulam, 1996). Furthermore, agonists and antagonists of acetylcholine receptors affect memory function, that is, they lead to the improvement and impairment of memory, respectively (Fitz, Gibbs, \& Johnson, 2008; Gold, 2003). Thus, the cholinergic system is also a research target of the cellular mechanism of memory functions. There are two types of acetylcholine receptor: muscarinic acetylcholine receptors (mAChR) and nicotinic acetylcholine receptors (nAChR). The activation of $\mathrm{mAChR}$ reportedly enhances the magnitude of LTP at SC-CA1 synapses in vivo and in vitro (Ovsepian, Anwyl, \& Rowan, 2004; Shinoe, Matsui, Taketo, \& Manabe, 2005); however, the mechanism by which it enhances synaptic plasticity remains unclear.

The inhibition of $\mathrm{K}^{+}$currents is one candidate because $\mathrm{mAChR}$ activation is known to inhibit some $\mathrm{K}^{+}$currents (Brown \& Adams, 1980; Higashida, Hoshi, Zhang, 
Yokoyama, Hashii, Jin, Noda, \& Robbins, 2005). The inhibition of $\mathrm{K}^{+}$channels can induce LTP. For example, the application of a blocker of $\mathrm{K}^{+}$channels, tetraethylammonium (TEA), induces LTP in all hippocampal subregions in vitro (Aniksztejn \& Ben-Ari, 1991; Coogan, O’Leary, \& O’Connor, 1999; Suzuki \& Okada, 2007). This form of LTP is independent of the activation of NMDA receptors, but dependent on voltagedependent $\mathrm{Ca}^{2+}$ channels (VDCC) instead.
The influx of $\mathrm{Ca}^{2+}$ via VDCC is a trigger for the induction of LTP as described above.

Recently, it was reported that the activation of mAChR enhanced NMDA receptor-mediated current via the inhibition of small conductance $\mathrm{K}^{+}$channels (SK channels) (Buchanan, Petrovic, Chamberlain, Marrion, \& Mellor, 2010). The enhancement of NMDA receptor-mediated current resulted in the enhancement of LTP. Another candidate is $\mathrm{Kv} 7 / \mathrm{M} \mathrm{K}^{+}$channels because the

\section{A Str. Ori. Stim.}

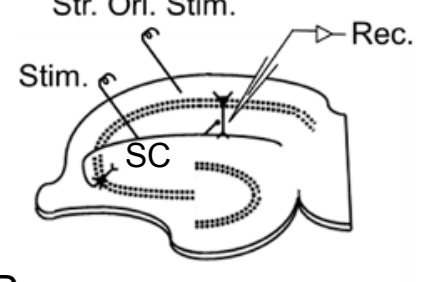

B
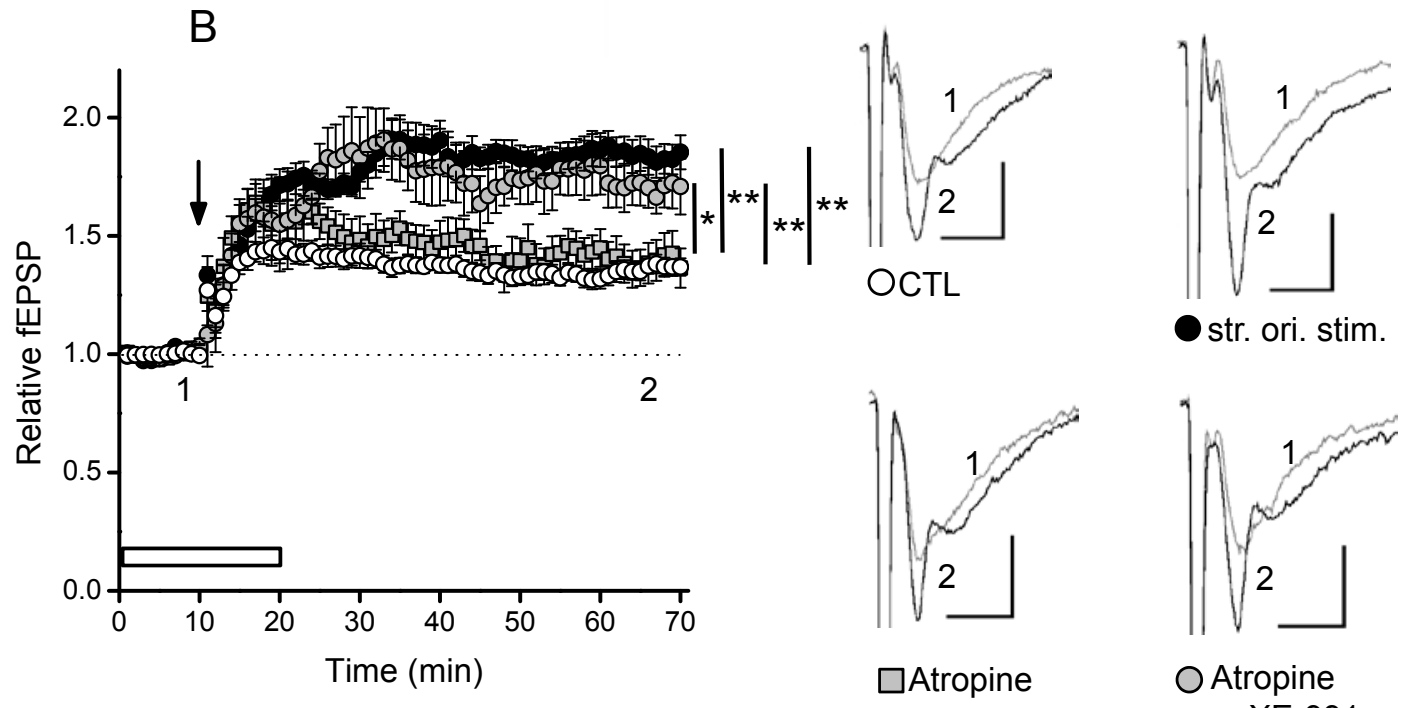

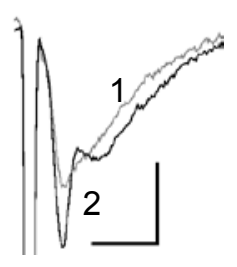

$\square$ Atropine

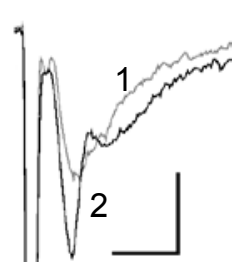

O Atropine

+ XE-991

Figure 1. The enhancement of LTP by cholinergic activation.

A: Schema of hippocampal slice and electrodes. Schaffer collaterals (SC) were stimulated every $10 \mathrm{~s}$ (Stim.) and field excitatory postsynaptic potentials (fEPSPs) were recorded from the CA1 stratum radiatum (Rec.). The $40-\mathrm{Hz}$ stimulation of the stratum oriens (str. ori. stim.) was applied $30 \mathrm{~s}$ before the tetanic stimulation of SC, when needed. B: Tetanic stimulation (arrow) of SC induced typical LTP (open circles). When a $40 \mathrm{~Hz}$ stimulus was applied to the stratum oriens, the magnitude of the LTP was enhanced (filled circles) $(\mathrm{p}<0.01$, compared with control). Bath application of the mAChR antagonist atropine $(10 \mu \mathrm{M})$ inhibited the enhancement of LTP by the stimulation of the stratum oriens (gray squares) ( $\mathrm{p}<0.01$, compared with str. ori. stim.). This atropine-induced inhibition was rescued by the co-application of the Kv7/M K${ }^{+}$channel blocker XE-991 $(10 \mu \mathrm{M})$ (gray circles) $(\mathrm{p}<0.01$, compared with control). The white bar indicates the timing of drug application. Insets: Typical waveforms of the synaptic responses obtained before (1) and $60 \mathrm{~min}$ after (2) the tetanic stimulation under each condition. The relative amplitudes of the fEPSP are shown as the mean \pm S.E. The traces were taken at the times indicated by the numbers in the figure. Scale: $5 \mathrm{~ms}, 0.5 \mathrm{mV}$. ${ }^{* *} \mathrm{p}<0.01,{ }^{*} \mathrm{p}<0.05$. 
activation of M1-type mAChR (M1 mAChR) has been shown to inhibit $\mathrm{Kv} 7 / \mathrm{M} \mathrm{K} \mathrm{K}^{+}$ channels (Delmas \& Brown, 2005; Higashida, Hoshi, Zhang, et al., 2005), and M1 mAChR is mainly involved in the facilitating effect of mAChR on LTP (Shinoe, Matsui, Taketo, et al., 2005). Thus, we electrophysiologically examined whether $\mathrm{Kv} 7 / \mathrm{M} \mathrm{K}^{+}$channels are involved in the facilitating effect of $\mathrm{mAChR}$ activation on tetanus-induced LTP in the rat hippocampal CA1 area (Suzuki \& Okada, 2012). This research is summarized in this paper.

Repetitive stimulation $(40 \mathrm{~Hz}, 0.5 \mathrm{~s})$ to the stratum oriens in CA1 was applied $30 \mathrm{~s}$ before the tetanic stimulation of the SC. The stratum oriens contains fibers from the medial septum. The magnitude of tetanusinduced LTP was enhanced when the stratum oriens was stimulated (Figure 1, filled circle). The enhancement of tetanusinduced LTP was blocked in the presence of the $\mathrm{mAChR}$ antagonist atropine (Figure 1, gray square). When inhibitor of the $\mathrm{Kv} 7 / \mathrm{M}$ $\mathrm{K}^{+}$channel, XE-991, was applied, the enhancement of tetanus-induced LTP was observed, even in the presence of atropine (Figure 1, gray circle). Furthermore, the application of $\mathrm{Kv} 7 / \mathrm{M} \mathrm{K}^{+}$channel inhibitor enhanced tetanus-induced LTP without stimulation of the stratum oriens (Figure 2, XE-991 w/o str. ori. stim.). When the stimulation of the stratum oriens was applied in the presence of XE-991, the magnitude of tetanus-induced LTP was not additionally enhanced. These results suggest that the activation of $\mathrm{mAChR}$ and the inhibition of the $\mathrm{Kv} 7 / \mathrm{M} \mathrm{K}^{+}$channel are involved in the enhancement of tetanus-induced LTP by the stimulation of the stratum oriens.

What is the intracellular mechanism behind the enhancement of tetanus-induced LTP by the activation of mAChR? The activation of $\mathrm{mAChR}$ activates an intracellular signaling cascade. One of the targets is phospholipase C (PLC). The activation of PLC makes diacylglycerol (DAG) and $\mathrm{IP}_{3}$ from PIP2. DAG activates the A-kinase anchoring protein (AKAP)-protein kinase $\mathrm{C}$ (PKC) complex, which results in inhibition of the $\mathrm{Kv} 7 / \mathrm{M} \mathrm{K}^{+}$channel. When PLC was inhibited by a selective blocker, U-73122, mAChR activation-induced enhancement of tetanusinduced LTP was blocked (Figure 2, U-73122). Thus, the activation of PLC is important for the enhancement of tetanus-induced LTP. As described above, while the influx of $\mathrm{Ca}^{2+}$ via NMDA receptors is crucial for LTP induction, tetanic stimulation with preceding stimulation of the stratum oriens induced

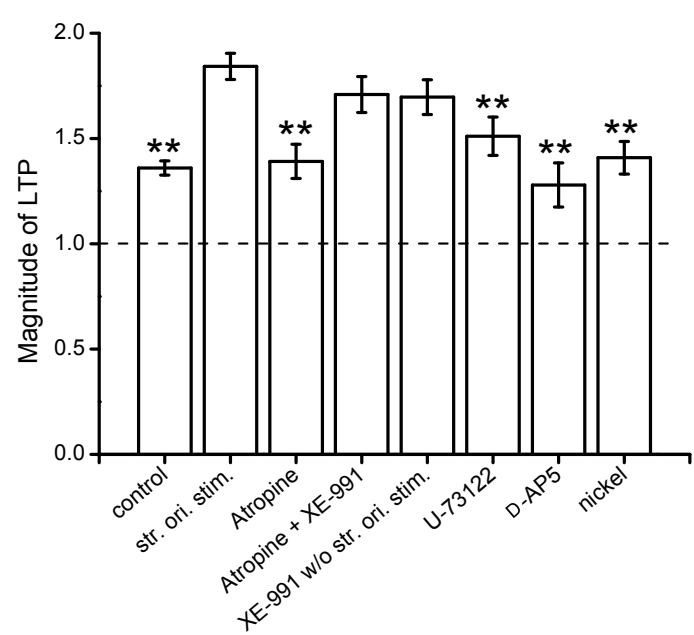

Figure 2. The magnitude of LTP in each experimental condition.

The magnitude of LTP 50-60 min after the tetanic stimulation was calculated. In control and XE-991 w/o str. ori. stim. conditions, tetanic stimulation was applied to the SC without str. ori. stim. In other conditions (str. ori. stim., Atropine, Atropine + XE-991, U-73122, D-AP5 and nickel), the stimulation of the stratum oriens was applied before the tetanic stimulation. The application of atropine inhibited the enhancement of tetanus-induced LTP by str. ori. stim. (Atropine). However, the application of $\mathrm{Kv} 7 / \mathrm{M} \mathrm{K}^{+}$channel inhibitor, XE-991, enhanced the magnitude of tetanus-induced LTP even in the presence of atropine or without str. ori. stim. (Atropine + XE-991 and XE-991 w/o str. ori. stim., respective1y). On the other hand, intracellular PLC blocker U-73122 and T/R-type voltage-dependent $\mathrm{Ca}^{2+}$ channel blocker nickel inhibited the enhancement of tetanus-induced LTP. Moreover, str. ori. stim. with tetanic stimulation induced LTP under the blockade of NMDA receptors (D-AP5). The magnitudes of LTP are shown as the mean \pm S.E. The statistical significances were calculated compared with str. ori. stim. ${ }^{* *} \mathrm{p}<0.01$. 


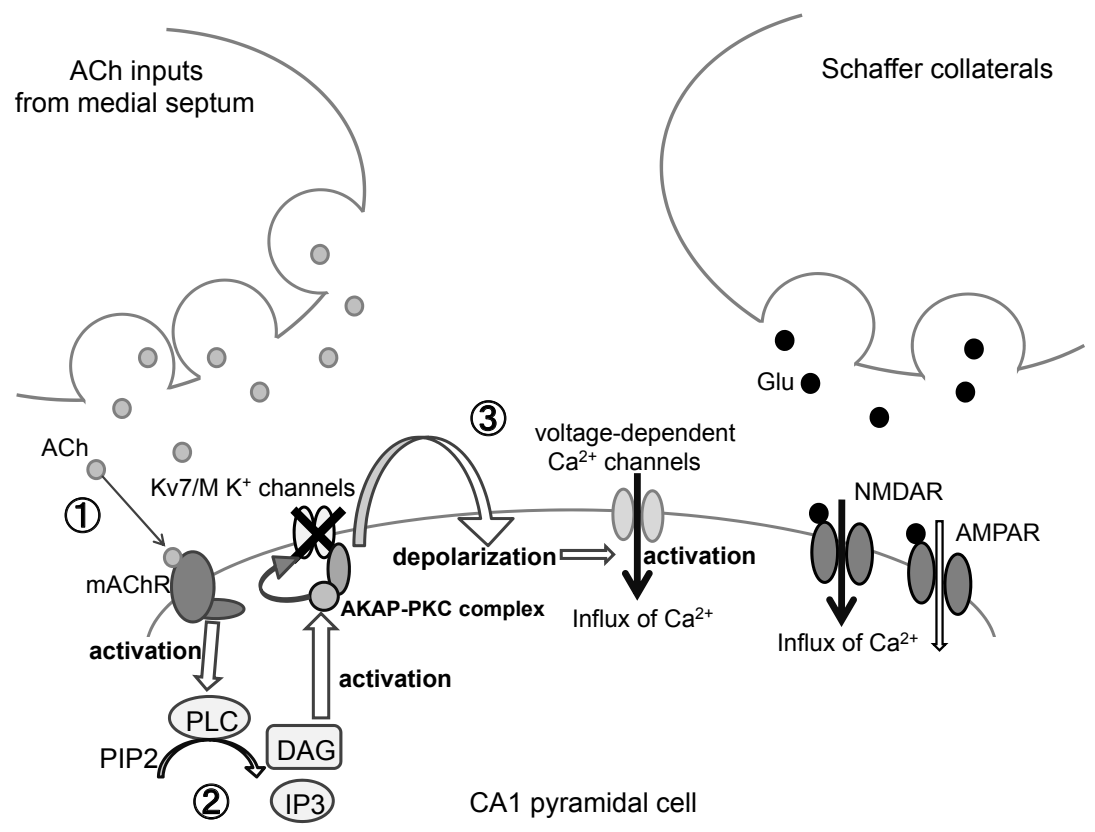

Figure 3. The proposed schema of the enhancement of tetanus-induced LTP by cholinergic modulation. (1) The stimulation of cholinergic input releases acetylcholine, and then muscarinic acetylcholine receptors (mAChRs) are activated. $\mathrm{mAChR}$ activates an intracellular signaling pathway that includes the phospholipase C (PLC) cascade. (2) The activation of PLC makes diacylglycerol (DAG) and IP3. DAG activates protein kinase $\mathrm{C}(\mathrm{PKC})$ and $\mathrm{Kv} 7 / \mathrm{M} \mathrm{K}^{+}$channels are inhibited. (3) The inhibition of $\mathrm{Kv} 7 / \mathrm{M} \mathrm{K}^{+}$channels results in the depolarization of cell membrane, and then voltage-dependent calcium channels are activated, which causes an influx of $\mathrm{Ca}^{2+}$.

LTP in the presence of NMDA receptor antagonist, D-AP5 (Figure 2, D-AP5). That is, the intracellular concentration of $\mathrm{Ca}^{2+}$ is increased by another mechanism. Because the influx of $\mathrm{Ca}^{2+}$ via VDCC is important for LTP induction by the $\mathrm{K}^{+}$channel blocker TEA, it is thought that the depolarization of the cell membrane by the inhibition of the $\mathrm{Kv} 7 / \mathrm{M} \mathrm{K}^{+}$channel might activate VDCC. Consistent with this hypothesis, the blockade of T/R-type VDCC prevented the mAChR activation-induced enhancement of tetanusinduced LTP (Figure 2, nickel). Taking these findings together, the stimulation of cholinergic input releases acetylcholine, and then mAChR is activated. mAChR activates an intracellular signaling pathway that includes the PLC cascade, and $\mathrm{Kv} 7 / \mathrm{M} \mathrm{K}^{+}$channels are inhibited. The inhibition of $\mathrm{Kv} 7 / \mathrm{M} \mathrm{K}^{+}$ channels results in depolarization of the cell membrane, and then VDCC is activated, which causes an influx of $\mathrm{Ca}^{2+}$ (Figure 3).
It is reported that the concentration of acetylcholine in the hippocampus is enhanced during the acquisition of operant behavior (Orsetti, Casamenti, \& Pepeu, 1996) and novel stimuli enhance acetylcholine release in the hippocampus (Acquas, Wilson, \& Fibiger, 1996). Therefore, the enhancement effect of LTP by mAChR activation might be important for memory acquisition.

\section{References}

Acquas, E., Wilson, C., \& Fibiger, H. C. 1996 Conditioned and unconditioned stimuli increase frontal cortical and hippocampal acetylcholine release: effects of novelty, habituation, and fear. Journal of Neuroscience, 16, 3089-3096.

Aniksztejn, L., \& Ben-Ari, Y. 1991 Novel form of long-term potentiation produced by a $\mathrm{K}^{+}$channel blocker in the hippocampus. Nature, 349, 67-69. 
SUZUKI : Cholinergic modulation for hippocampal long-term potentiation

Bliss, T. V. P., \& Collingridge, G. L. 1993 A synaptic model of memory: long-term potentiation in the hippocampus. Nature, 361, 31-39.

Brown, D. A., \& Adams, P. R. 1980 Muscarinic suppression of a novel voltage-sensitive $\mathrm{K}^{+}$current in a vertebrate neurone. Nature, 283, 673-676.

Buchanan, K. A., Petrovic, M. M., Chamberlain, S. E., Marrion, N. V., \& Mellor, J. R. 2010 Facilitation of long-term potentiation by muscarinic $\mathrm{M}_{1}$ receptors is mediated by inhibition of SK channels. Neuron, 68, 948-963.

Davies, P., \& Maloney, A. J. 1976 Selective loss of central cholinergic neurons in Alzheimer's disease. Lancet, 2, 1403.

Fitz, N. F., Gibbs, R. B., \& Johnson, D. A. 2008 Selective lesion of septal cholinergic neurons in rats impairs acquisition of a delayed matching to position T-maze task by delaying the shift from a response to a place strategy. Brain Research Bulletin, 77, 356-360.

Geula, C., \& Mesulam, M. M. 1996 Systematic regional variations in the loss of cortical cholinergic fibers in Alzheimer's disease. Cerebral Cortex, 6, 165-177.

Gold, P. E. 2003 Acetylcholine modulation of neural systems involved in learning and memory. Neurobiology of Learning and Memory, 80, 194-210.

Higashida, H., Hoshi, N., Zhang, J. S., Yokoyama, S., Hashii, M., Jin, D., Noda, M., Robbins, J. 2005 Protein kinase C bound with A-kinase anchoring protein is involved in muscarinic receptor-activated modulation of M-type KCNQ potassium channels. Neuroscience Research, 51, 231234.

Orsetti, M., Casamenti, F., \& Pepeu, G. 1996 Enhanced acetylcholine release in the hippocampus and cortex during acquisition of an operant behavior. Brain Research, 724, 89-96.

Ovsepian, S. V., Anwyl, R., \& Rowan, M. J. 2004 Endogenous acetylcholine lowers the threshold for long-term potentiation induction in the CA1 area through muscarinic receptor activation: in vivo study. European Journal of Neuroscience, 20, $1267-1275$.

Shinoe, T., Matsui, M., Taketo, M. M., \& Manabe, T. 2005 Modulation of synaptic plasticity by physiological activation of M1 muscarinic acetylcholine receptors in the mouse hippocampus. Journal of Neuroscience, 25, 11194-11200.

Suzuki, E., \& Okada, T. 2007 Regional differences in GABAergic modulation for TEA-induced synaptic plasticity in rat hippocampal CA1, CA3 and dentate gyrus. Neuroscience Research, 59, 183-190.

Suzuki, E., \& Okada, T. 2012 Stratum oriens stimulation-evoked modulation of hippocampal long-term potentiation involves the activation of muscarinic acetylcholine receptors and the inhibition of $\mathrm{Kv} 7 / \mathrm{M}$ potassium ion channels. European Journal of Neuroscience, 36, 1984-1992.

(2014. 4.30 受稿, 2014. 5 . 31 受理) 